Nota Científica

\title{
Espécies florestais e formigas cortadeiras (Hymenoptera: Formicidae) em Viçosa, Minas Gerais
}

\author{
Alexandre Arnhold ${ }^{1}$, Iris Cristiane Magistrali ${ }^{2}$, Norivaldo dos Anjos ${ }^{3}$ \\ ${ }^{1}$ Universidade Federal de Lavras, Departamento de Entomologia, Campus Universitário, s/n, CEP 37200-000, Lavras, MG, Brasil \\ ${ }^{2}$ Universidade Federal de Santa Maria, Centro de Ciências Rurais, Departamento de Defesa Fitossanitária, Campus Universitário, Av. Roraima, s/n, \\ CEP 97105-900, Santa Maria, RS, Brasil \\ ${ }^{3}$ Universidade Federal de Viçosa, Departamento de Entomologia, Casa dos Cupins, Av. P. H. Rolfs, s/n, CEP 36570-000, Viçosa, MG, Brasil
}

"Autor correspondente:

alexarnhold@yahoo.com.br

Termos para indexação:

Entomologia florestal

Ninhos

Ritmo diário de forrageamento

\section{Index terms:}

Forest entomology

Nests

Diel patterns of foraging

Histórico do artigo:

Recebido em 07/03/2012

Aprovado em 21/02/2013

Publicado em 28/06/2013

doi: 10.4336/2013.pfb.33.74.377

\begin{abstract}
Resumo - O objetivo deste trabalho é ampliar os registros de espécies florestais atacadas por formigas cortadeiras no município de Viçosa, Estado de Minas Gerais. Este trabalho foi realizado no Campus da Universidade Federal de Viçosa, onde se buscou formigueiros ativos que estavam cortando e carregando fragmentos de essências florestais. Foram tomadas as medidas da temperatura e umidade relativa do ar, as distâncias do olheiro ativo e do ninho até a árvore atacada e ainda o comprimento da trilha de forrageamento. Com os resultados obtidos observa-se a ocorrência de três espécies de quenquém Acromyrmex niger, A. subterraneus molestans e A. subterraneus subterraneus e duas espécies de saúva Atta sexdens rubropilosa e A. laevigata injuriando 14 espécies florestais.
\end{abstract}

\section{Forest cultures and leaf cutting ants in Viçosa, State Minas Gerais, Brazil}

\begin{abstract}
This study aimed to enlarge the records of forest species attacked by leaf cutting ants in Viçosa, in the state of Minas Gerais. The rising was accomplished in the Campus of the Universidade Federal de Viçosa, being considered only nest active that were cutting and carrying fragments of forest essences. For the study it was evaluated temperature measures and distances of the nest to the attacked tree. As results are observed the occurrence of three quenquém species (Acromyrmex niger Smith, F., 1858, Acromyrmex subterraneus molestans Santschi, 1925 and Acromyrmex subterraneus subterraneus Forel, 1893) and two saúva species (Atta sexdens rubropilosa Forel, 1908 and A. laevigata F. Smith, 1858) attacking 14 forest species.
\end{abstract}

As formigas cortadeiras possuem o hábito de cortar e carregar pedaços de vegetais para dentro de seus ninhos, para servir de substrato para um fungo do qual se alimentam. Dentre as espécies mais importantes de formigas cortadeiras, destacam-se as saúvas e as quenquéns, respectivamente dos gêneros Atta e Acromyrmex (Dela Lucia et al., 2000). Loureiro \& Queiroz (1990) relataram a ocorrência dos gêneros Atta e Acromyrmex no município de Viçosa, MG. Para o gênero Atta, ocorrem as espécies A. bisphaerica
Forel, 1908 (Della Lucia et al., 1992; Araújo, 2003), A. sexdens rubropilosa Forel, 1908 (Vanetti, 1957; Silva, 2001; Anjos et al., 2008) e, também, A. laevigata F. Smith, 1858 (Rezende \& Della Lucia, 1999). Já para o gênero Acromyrmex, há registros para a ocorrência de A. subterraneus molestans Santschi, 1925 (Souza et al., 2004; Anjos et al., 2008), A. subterraneus subterraneus Forel, 1893 (Della Lucia et al., 1995; Rezende \& Della Lucia, 1999; Anjos et al., 2008) e, ainda, de A. niger Smith, F., 1858 (Anjos et al., 2008). 
Não obstante a quantidade de trabalhos, poucos se referem às plantas forrageadas.

O objetivo deste trabalho foi o de ampliar os registros de ataque de formigas cortadeiras em diferentes espécies florestais.

O levantamento das formigas cortadeiras, em atividade de forrageamento sobre espécies florestais, foi realizado a uma latitude de $20^{\circ} 46^{\prime} 44^{\prime \prime} \mathrm{S}$, longitude de $42^{\circ} 52^{\prime} 55^{\prime}$ 'O, altitude de 650 metros. Foram coletadas somente formigas que estavam forrageando em espécies florestais produtoras de madeira ou de uso paisagístico. A coleta foi realizada, manualmente, no campus da Universidade Federal de Viçosa, MG, em árvores de diversas idades, isoladas ou não, no período de 10 a 28 de março de 2008, das 08:00 às 18:00 horas. No mês de março inicia-se o outono e a temperatura começa a diminuir na região. Segundo Robinson \& Fowler (1982), a temperatura e a umidade do ar tem influência direta na atividade das formigas. Nos casos em que o formigueiro estava em plena atividade de forrageamento, mediram-se a umidade relativa e a temperatura do ar, a $2 \mathrm{~m}$ acima do solo, utilizando-se de um termohigrômetro da marca HAAR-SYNTH.HYRO, com escala de 0 a $100 \%$ e precisão de $1 \%$ para a umidade relativa e escala de $-15{ }^{\circ} \mathrm{C}$ a $55{ }^{\circ} \mathrm{C}$ e precisão de $1{ }^{\circ} \mathrm{C}$ para a temperatura ambiente. As distâncias da árvore atacada até o olheiro ativo e até o ninho, bem como o comprimento da trilha, foram medidas utilizando-se trena centimétrica. As espécies das formigas encontradas foram determinadas segundo Mayhé-Nunes (1991).

Foram encontrados 18 formigueiros ativos, pertencentes a três espécies de Acromyrmex e a duas de Atta, forrageando em 18 hospedeiros de 14 espécies florestais diferentes (Tabela 1).

Apesar do esforço, em dois casos foi impossível localizar o formigueiro devido à falta de trilha, à presença de capinzais e à de galhadas no local. Isto significa que nem sempre é possível combater diretamente todos os formigueiros de uma região, em função deste tipo de dificuldade. Dos formigueiros em atividade, 12 pertenciam ao gênero Acromyrmex e seis ao gênero Atta. As formigas encontradas forrageando as

Tabela 1. Formigas cortadeiras injuriando espécies florestais em Viçosa, Minas Gerais, no período de10 a 28 de março de 2008.

\begin{tabular}{|c|c|c|c|c|c|c|c|}
\hline $\begin{array}{l}\text { Espécie de formiga } \\
\text { Cortadeira }\end{array}$ & $\begin{array}{c}\text { Hospedeiro } \\
\text { Florestal }\end{array}$ & $\begin{array}{c}\mathrm{T} \\
\left({ }^{\circ} \mathrm{C}\right)\end{array}$ & $\begin{array}{l}\text { UR } \\
(\%)\end{array}$ & $\begin{array}{l}\text { Horário de } \\
\text { avaliação }\end{array}$ & $\begin{array}{c}\text { DAO } \\
\text { (m) }\end{array}$ & $\begin{array}{c}\text { DAN } \\
(\mathbf{m})\end{array}$ & $\begin{array}{l}\text { CT } \\
\text { (m) }\end{array}$ \\
\hline \multirow{6}{*}{ Acromyrmex Niger } & Ligustrum japonicum Thunb. & 30 & 62 & $10: 25$ & 1,20 & -- & 1,47 \\
\hline & Prunus sellowii Koehne & 28 & 79 & $10: 40$ & 1,70 & 1,70 & 1,20 \\
\hline & $\begin{array}{l}\text { Callistemon linearis (Schrad. \& } \\
\text { J.C.Wendl.) Sweet }\end{array}$ & 30 & 65 & $15: 30$ & 0,80 & 0,80 & 0,90 \\
\hline & Eucalyptus grandis Hill ex Maiden & 30 & 70 & $10: 10$ & -- & -- & -- \\
\hline & Caesalpinia peltophoroides Benth & 29 & 65 & $13: 45$ & 1,60 & 1,60 & 1,75 \\
\hline & Eucalyptus urophylla S.T. Blake & 28 & 77 & $11: 20$ & 3,00 & 3,00 & 3,20 \\
\hline \multirow{3}{*}{$\begin{array}{l}\text { Acromyrmex } \\
\text { subterraneus } \\
\text { subterraneus }\end{array}$} & Michelia champaca L. & 29 & 59 & $15: 10$ & 0,52 & 0,52 & 0,55 \\
\hline & Delonix regia (Bojer ex Hook.) & 29 & 59 & $15: 30$ & 0,80 & 0,80 & 0,90 \\
\hline & Dombeya wallichii (Lindl.) Benth. & 31 & 60 & $14: 30$ & 3,80 & 3,80 & 4,70 \\
\hline \multirow{3}{*}{$\begin{array}{c}\text { Acromyrmex } \\
\text { subterraneus } \\
\text { molestans }\end{array}$} & Spathodea campanulata Beauv. & 30 & 58 & $15: 00$ & 1,20 & 1,30 & 1,45 \\
\hline & Spathodea campanulata Beauv. & 29 & 59 & $15: 35$ & 0,20 & 0,20 & 1,60 \\
\hline & Delonix regia (Bojer ex Hook.) & 29 & 61 & $15: 45$ & 14 & 14 & 15 \\
\hline \multirow{5}{*}{$\begin{array}{l}\text { Atta sexdens } \\
\text { rubropilosa }\end{array}$} & Sterculia chicha St. Hil. & 30 & 60 & $8: 50$ & 2,28 & 4,00 & 2,90 \\
\hline & Eucalyptus grandis Hill. ex Maiden & 31 & 69 & $10: 30$ & 4,10 & 5,00 & 4,60 \\
\hline & Albizia lebbeck (L.) Benth. & 30 & 60 & $13: 00$ & 6,50 & 8,00 & 11,70 \\
\hline & Hibiscus rosa-sinensis $\mathrm{L}$. & 30 & 60 & $13: 15$ & 3,50 & 8,00 & 3,70 \\
\hline & Caesalpinia peltophoroides Benth. & 29 & 65 & $13: 30$ & 0,10 & 1,00 & 0,10 \\
\hline Atta laevigata & Mabea fistulifera Mart. & 27 & 80 & $9: 40$ & 3,00 & 11 & 8,00 \\
\hline
\end{tabular}

Em que: $\mathrm{T}=$ temperatura; $\mathrm{DAO}=$ Distância da árvore ao olheiro ativo; $\mathrm{DAN}=$ Distância da árvore ao ninho; $\mathrm{CT}=\mathrm{Comprimento} \mathrm{da} \mathrm{trilha.}$ 
diferentes essências florestais foram: Acromyrmex niger, Acromyrmex subterraneus subterraneus, Acromyrmex subterraneus molestans, Atta sexdens rubropilosa e Atta laevigata.

No presente trabalho observou-se predominância do gênero Acromyrmex, representando 66,6\% dos casos. Em um trabalho semelhante, realizado entre os meses de agosto e setembro, Anjos et al. (2008) encontraram uma predominância de 89,5\% desse gênero em relação à de Atta. Entretanto, trabalhos que relacionem a atividade de forrageamento com as condições climáticas na área estudada são escassos na literatura.

Silva et al. (1968) registraram ocorrência de Acromyrmex niger nos estados de MG, SP, CE, RJ, ES, PR e SC. Em Minas Gerais, Pacheco \& Berti Filho (1987) relataram sua ocorrência no vale do Rio Doce e Gonçalves (1961) nos municípios de Belo Horizonte, Ouro Preto, João Monlevade, Barbacena, Palmira e Viçosa. Pacheco \& Berti Filho (1987) relataram esta espécie de formiga cortadeira atacando várias espécies de eucalipto, no município de Aurora, SP. Carvalho (1950) registrou ataques de A niger sobre Araucaria angustifolia (Bertol.) O. Kuntze, em Canoinhas, SC. Em Viçosa, Anjos et al. (2008) registraram a ocorrência de $A$. niger forrageando em nove diferentes espécies florestais; entre elas, Ligustrum japonicum e Eucalyptus grandis foram também aqui observadas. Neste levantamento se constatou o ataque de A. niger em árvores de Prunus sellowii, Callistemon linearis e em Caesalpinia peltophoroides.

É importante salientar, a exemplo do que foi encontrado por Anjos et al. (2008), que A. niger foi a espécie de formiga cortadeira que forrageava o maior número de espécies arbóreas, representando 27,78\% dos casos.

Segundo Della Lucia et al. (2000), a espécie Acromyrmex subterraneus subterraneus se encontra distribuída nos estados do Amazonas, Ceará, Rio Grande do Norte, Mato Grosso, Minas Gerais, Rio de Janeiro, Paraná, Santa Catarina, Rio Grande do Sul e São Paulo. No estado de Minas Gerais, a ocorrência desta espécie já foi relatada em Belo Horizonte, Ouro Preto, Palmira, João Monlevade, Barbacena e Viçosa por Gonçalves (1961). Registro do ataque desta formiga foi feito por Rezende \& Della Lucia (1999) em Corymbia citriodora Hill \& Johnson, no município de Coimbra, MG. Observou-se pela primeira vez neste trabalho o ataque de Acromyrmex subterraneus subterraneus a espécies florestais no município de Viçosa e também o ataque de formigas cortadeiras a Michelia champaca e Dombeya wallichii.

A ocorrência de A. subterraneus molestans em Viçosa, MG, já tinha sido relatada por Della Lucia et al. (1995) e por Rezende \& Della Lucia (1999).

A Atta sexdens rubropilosa é uma espécie de formiga que já foi registrada em GO, MA, MG, MT, PR, DF, SP e MS por Gonçalves (1942 e 1945), PR por Paula (1956), MG por Oliveira (1996), DF, GO, MA, MG, MT, PR, SP e MS por Rando (2002), cortando folhas de mais de 30 espécies diferentes de essências florestais. Conforme afirmou Silva (2001), esta formiga cortadeira já tinha sido observada no município de Viçosa, MG, onde causava danos a Hevea brasiliensis Mull Arg., mas Anjos et al. (2008) também a encontraram neste município forrageando em Eucalyptus grandis e em Anadenanthera macrocarpa (Benth.) Brenan. Observouse também neste levantamento o ataque de $A$. sexdens rubropilosa a Sterculia chicha, Albizia lebbeck, Hibiscus rosa-sinensis e Caesalpinia peltophoroides.

Atta laevigata é a segunda espécie de formiga cortadeira mais comum no Brasil, tendo ampla distribuição pelo interior do país (Anjos et al., 1998). No estado de Minas Gerais, A. laevigata já foi registrada ocorrendo em plantações de eucaliptos em Curvelo (Zanuncio et al., 1999) e em João Pinheiro (Grandeza et al., 1999). Segundo Peternelli et al. (2004), as formigas de A. laevigata têm o hábito de carregarem sementes de Mabea fistulifera, mas no presente trabalho, elas estavam apenas cortando e carregando pedaços de flores e de folhas.

Para os 18 formigueiros encontrados, a distância entre a árvore atacada e o olheiro ativo foi de 2,8 $\pm 0,9 \mathrm{~m}$; da árvore ao ninho foi $4,0 \pm 1,0 \mathrm{~m}$ e o comprimento da trilha foi de $3,7 \pm 1,0 \mathrm{~m}$. O comprimento da trilha para as espécies de quenquém foi de $3,7 \pm 1,3 \mathrm{~m}$, enquanto para as de saúva foi de 5,1 $\pm 1,6 \mathrm{~m}$. A distância da árvore ao ninho foi de $3,2 \pm 0,8 \mathrm{~m}$ para quenquém e de $6,2 \pm 1,4 \mathrm{~m}$ para saúva. A distância da árvore ao olheiro ativo foi de 2,9 $\pm 1,1 \mathrm{~m}$ para quenquéns e $3,2 \pm 1,2 \mathrm{~m}$ para saúvas.

A temperatura, nos horários de forrageamento, variou entre a mínima de $27{ }^{\circ} \mathrm{C}$ e a máxima de $31{ }^{\circ} \mathrm{C}$, já a umidade relativa variou entre 58 e $80 \%$. A temperatura e a umidade relativa para o período de estudo foram $29,4 \pm 1,0{ }^{\circ} \mathrm{C}$ e $64,9 \pm 1,7 \%$. As médias nos últimos dez anos, da temperatura e da umidade relativa do ar, para o mês de março, em Viçosa, $\mathrm{MG}$, foram de $22{ }^{\circ} \mathrm{C}$ e $82 \%$, 
respectivamente. Portanto, a temperatura média durante o período de estudo foi mais elevada do que a média dos últimos dez anos para esta mesma data; a umidade relativa foi mais baixa.

Os horários predominantes de forrageamento foram os vespertinos representando $61 \%$ dos casos observados, o que contradiz o trabalho realizado por Poderoso et al. (2007).

Robinson \& Fowler(1982) mencionaram a temperatura como o principal agente de modificação no horário da atividade forrageadora em formigas cortadeiras. Araújo (1996) observou que o aumento da temperatura provoca uma diminuição na atividade de forrageamento de Acromyrmex laticeps nigrosetosus Forel, 1908. Assim, pode-se inferir que a maior atividade de forrageamento na parte da manhã foi devido a uma inibição desta atividade causada pela elevação da temperatura na parte da tarde.

Foram encontradas 14 diferentes espécies florestais sendo atacadas. Este é o primeiro registro do ataque de Acromyrmex niger em Prunus sellowii, Callistemon linearis e em Caesalpinia peltophoroides, no Brasil; o primeiro registro do ataque de Acromyrmex subterraneus subterraneus a espécies florestais no município de Viçosa; Michelia champaca e Dombeya wallichii, são registradas pela primeira vez sendo atacadas por formigas cortadeiras no Brasil; e para Sterculia chicha, Albizia lebbeck, Hibiscus rosa-sinensis e Caesalpinia peltophoroides, este é o primeiro registro de ataque de Atta sexdens rubropilosa, no Brasil.

\section{Referências}

ANJOS, N.; ARNHOLD, A.; CORRÊA, G. V. V.; STUMPF, K. Árvores e formigas cortadeiras (Hymenoptera: Formicidae) em Viçosa, Minas Gerais. Revista Trópica, Chapadinha, v. 2, n. 1, p. 11-16, 2008

ANJOS, N.; DELLA LUCIA, T. M. C.; MAYHÉ-NUNES, A. J. Guia prático sobre formigas cortadeiras em reflorestamentos. Ponte Nova: Graff Cor, 1998. 100 p.

ARAÚJO, M. S. Espécies de formigas cortadeiras e ecologia de Acromyrmex laticeps nigrosetosus num povoamento de eucalipto. 1996. 51 f. Dissertação (Mestrado em Entomologia) - Universidade Federal de Viçosa, Viçosa, MG.

ARAÚJO, M. S. O fogo como agente de distúrbio em comunidades de formigas. 2003. $81 \mathrm{f}$. Tese (Doutorado em Entomologia) - Universidade Federal de Viçosa, Viçosa, MG.
CARVALHO, A. L. Contribuição ao estudo da biologia na estação florestal dos pardos. Anuário Brasileiro de Economia Florestal, Rio de Janeiro, v. 3, n. 3. p. 208-222, 1950.

DELlA LUCIA, T. M. C.; CAMERON, R. S.; VILELA, E. F.; BENTO, J. M. S. Aceitação de iscas granuladas com sulfluramida, um novo princípio ativo, por formigas cortadeiras, no campo. Revista Árvore, Viçosa, MG, v. 16, n. 2, p. 218-223, 1992.

DELLA LUCIA, T. M. C.; OLIVEIRA, M. A.; ARAÚJO, M. S.; VILELA, E. F. Avaliação da não-preferência da formiga cortadeira Acromyrmex subterraneus subterraneus Forel ao corte de Eucalyptus. Revista Árvore, Viçosa, MG, v. 19, n.1, p. 92-99, 1995.

DELLA LUCIA, T. M. C.; ANJOS, N.; ZANUNCIO, J. C. Controle de formigas cortadeiras. Viçosa, MG: CTP, 2000. 52 p.

GONÇALVES, C. R. Contribuição para o conhecimento do gênero Atta Fabr., das formigas saúvas. Boletim da Sociedade Brasileira de Agronomia, Rio de Janeiro, v. 5, n. 3, p. 333- 358, 1942. GONÇALVES, C. R. Saúvas do Sul e Centro do Brasil. Boletim Fitossanitário, Rio de Janeiro, v. 2, n. 3-4, p. 183-218, 1945.

GONÇALVES, C. R. O gênero Acromyrmex no Brasil (Hymenoptera: Formicidae). Studia Entomológica, Petrópolis, v. 4, n. 1, p. 113-180, 1961

GRANDEZA, L. A. O.; MORAIS, J. C.; ZANETTI, R. Estimativa do crescimento externo de ninhos de Atta sexdens rubropilosa Forel e Atta laevigata (F. Smith) (Hymenoptera:Formicidae) em áreas de reflorestamento com eucalipto. Anais da Sociedade Entomológica do Brasil, Londrina, v. 28, n. 1, p. 59-64, 1999.

LOUREIRO, M. C.; QUEIROZ, M. V. de. Insetos de Viçosa: formicidae. Viçosa, MG: Ed. da UFV, 1990. 106 p.

MAYHÉ-NUNES, A. J. Estudo de Acromyrmex (Hymenoptera: Formicidae) com ocorrência constatada no Brasil: subsídios para uma análise filogenética. 1991. 122 f. Dissertação (Mestrado em Entomologia) - Universidade Federal de Viçosa, Viçosa, MG.

OLIVEIRA, M. A. Identificação de formigas cortadeiras e efeito do desfolhamento simulado em plantios de Eucalyptus grandis. 1996. 67 f. Dissertação (Mestrado em Entomologia) - Universidade Federal de Viçosa, Viçosa, MG.

PACHECO, P.; BERTI FILHO, E. (Ed.) Formigas cortadeiras e o seu controle. Piracicaba, SP: IPEF, 1987. 152 p.

PAULA, H. S. Ocorrência de saúvas no Estado do Paraná. Boletim Fitossanitário, v. 6, n. 3-4, p. 153-158, 1956.

PETERNELLI. E. F. O. de; ELLA LUCIA, T. M. C.; MARTINS, S V. Espécies de formigas que interagem com as sementes de Mabea fistulifera Mart. (Euphorbiaceae). Revista Árvore, Viçosa, MG, v. 28, n. 5, p. 733-738, 2004.

PODEROSO, J. C. M.; GONÇALVES, F. B.; CORREIA-OLIVEIRA, M. E.; DANTAS, P. C.; OLIVEIRA, P. D. M.; GONÇALVES, G. B.; RIBEIRO, G. T. Atividade de forrageamento de Acromyrmex landolti balzani Emery, 1980 (Hymenoptera: Formicidae), no Campus da Universidade Federal de Sergipe, Brasil. In: CONGRESSO DE ECOLOGIA DO BRASIL, 8., 2007, Caxambu. Resumos... Caxambu: Sociedade de Ecologia do Brasil, 2007. p. 1-2. 
RANDO, J. S. S. Ocorrência de espécies de Atta Fabricius, 1804 e Acromyrmex Mayr, 1865 em algumas regiões do Brasil. 2002. 105 f. Tese (Doutorado Agronomia e Proteção de Plantas) - Universidade Estadual Paulista "Júlio Mesquita Filho", Botucatu.

REZENDE, M. A. A.; DELLA LUCIA, T. M. C. Is Eucalyptus citriodora attacked by leaf-cutting ants. Revista Árvore, Viçosa, MG, v. 23, n. 1, p. 69-74, 1999.

ROBINSON, S. W.; FOWLER, H. G. Foraging and pest potential of Paraguayan grass-cutting ants (Atta and Acromyrmex) to the cattle industry. Zeitschrift fuer Angewandte Entomologie, Hamburg, v. 93, p. 42-54, 1982.

SILVA, A. G. A.; GONÇALVES, C, R.; GALVÃO, D. M.; GONÇALVES, A. J. L.; GOMES, J.; SILVA, M. N.; SIMONI, L. Quarto catálogo dos insetos que vivem nas plantas do Brasil: seus parasitos e predadores: parte 2, tomo 1. Rio de Janeiro: Ministério da Agricultura, 1968. $622 \mathrm{p}$.
SILVA, E. J. Identificação de formigas cortadeiras e efeito do desfolhamento simulado em plantios de seringueira (Hevea brasiliensis Mull Arg.). 2001. 43 f. Dissertação (Mestrado em Entomologia) - Universidade Federal de Viçosa, Viçosa, MG.

SOUZA, D. J.; LINO NETO, J.; DELLA LUCIA, T. M. C.; PETERNELLI, E. Occurrence of polygyny em Acromyrmex subterraneus molestans Santschi, 1925 (Hymenoptera: Formicidae). Ciência Rural, Santa Maria, RS, v. 34, n. 5, p. 1611-1613, 2004.

VANETTI, F. Resultados dos tratamentos de sauveiros no período de 1949 a 1958. 1957. Revista Ceres, Viçosa, MG, v. 10, n. 57, p. 149-163, 1957.

ZANUNCIO, J. C.; PEREIRA J. M. M.; OLIVEIRA, H. N. de. Controle de Atta laevigata (Hymenoptera: Formicidae) com a isca Landrin-f, em área anteriormente coberta com Eucalyptus. Ciência Rural. Santa Maria, RS, v. 29, n. 4, p. 573- 576, 1999. 
